\title{
Itinerários terapêuticos: o estado da arte da produção científica no Brasil
}

\author{
Therapeutic itineraries: \\ state of the art of scientific production in Brazil
}

\author{
Ana Lucia Lobo Vianna Cabral ${ }^{1}$ \\ Angel Martinez-Hemáez ${ }^{2}$ \\ Eli Iola Gurgel Andrade ${ }^{3}$ \\ Mariangela Leal Cherchiglia ${ }^{3}$
}

${ }^{1}$ Universidade Federal de Minas Gerais - UFMG allcabral@yahoo.com.br ${ }^{2}$ Departamento de Antropologia, Universitat Rovira i Virgili (Tarragona, Espanha)

${ }^{3}$ Grupo de Pesquisa em

Economia da Saúde,

Departamento de Medicina

Preventiva e Social,

Faculdade de Medicina,

UFMG
Abstract Knowledge about the itineraries of people seeking healthcare may contribute to our understanding of behavior in relation to healthcare and utilization of health services. In Brazil, however, despite their relevance there have been few studies into healthcare-seeking behavior and those available are relatively new and have not received the attention they deserve from health researchers and managers. This article presents the results of a survey of the Brazilian scientific literature on this subject published in the past 20 years, describing and analyzing the approaches used. Data were collected between September and November 2008 through the portal of the Virtual Health Library. The search located 11 articles and content analysis was used to generate categories. The majority of these studies are grounded in socio-anthropological theory, and the main focus of interest is the patient's perception of disease and treatment. Only a few of these publications address patients' access to and utilization of health services. It is argued that the study of healthcareseeking behavior can be an important tool for improving the quality of healthcare.

Key words Anthropology, Helthcare-Seeking behavior, Accessibility of health services
Resumo O conhecimento sobre os itinerários de pessoas em busca de atenção à saúde pode contribuir para compreensão sobre o comportamento em relação ao cuidado e utilização de serviços de saúde. Apesar de sua potencialidade, os estudos sobre itinerários terapêuticos não tem expressão conhecida no Brasil. Este artigo busca apresentar um levantamento da produção científica nacional sobre o assunto nos últimos 20 anos, descrevendo e analisando as abordagens realizadas. Os dados foram coletados durante os meses de setembro e novembro de 2008 através do portal da Biblioteca Virtual em Saúde. A busca resultou em 11 artigos que foram submetidos à análise de conteúdo por meio de categorização. Observou-se que os estudos sobre itinerários terapêuticos no Brasil são recentes e pouco explorados por pesquisadores e gestores. A abordagem socioantropológica oferece suporte teórico à maioria dos estudos realizados. O principal foco de interesse é a percepção do paciente sobre a doença e tratamento. Poucos são os estudos que nessa discussão associam aspectos sobre o acesso e utilização dos serviços e fatores relacionados ao contexto do paciente. Conclui-se que o estudo sobre itinerários terapêuticos pode ser uma importante ferramenta para a qualificação da assistência.

Palavras-chave Antropologia, Comportamento de procura de cuidados de saúde, Acesso aos serviços de saúde 


\section{Introdução}

A preocupação sobre como e em que momento as pessoas procuram ajuda para resolver suas demandas ou problemas de saúde, tem estado cada vez mais presente em estudos sobre planejamento, organização e avaliação de serviços assistenciais de saúde ${ }^{1-3}$. A intenção é subsidiar a escolha de estratégias adequadas que garantam acesso aos usuários em momento oportuno e de forma contínua, propiciando vínculo com a equipe de profissionais de saúde e, consequentemente, adesão ao tratamento proposto.

Não obstante este desejo de gestores e trabalhadores de serviços de saúde, os caminhos percorridos por pessoas em busca de cuidados terapêuticos não necessariamente coincidem com esquemas ou fluxos pré-determinados. Suas escolhas expressam construções subjetivas individuais e também coletivas acerca do processo de adoecimento e de formas de tratamento, forjadas sob as influências de diversos fatores e contextos ${ }^{4}$. Estas escolhas vão definir ações que, passo a passo, constituirão um determinado percurso.

A literatura socioantropológica utiliza o termo itinerário terapêutico para definir este percurso. Segundo alguns autores, itinerários terapêuticos são constituídos por todos os movimentos desencadeados por indivíduos ou grupos na preservação ou recuperação da saúde, que podem mobilizar diferentes recursos que incluem desde os cuidados caseiros e práticas religiosas até os dispositivos biomédicos predominantes (atenção primária, urgência, etc. $)^{5}$. Referem-se a uma sucessão de acontecimentos e tomada de decisões que, tendo como objeto o tratamento da enfermidade, constrói uma determinada trajetória ${ }^{5}$.

Alves e Souza ${ }^{4}$, em revisão da literatura sobre "busca de atenção" localizam o surgimento das primeiras reflexões acerca de itinerários terapêuticos, atreladas ao estudo sobre o "comportamento do enfermo" (illness behavior), termo criado por Mechanic \& Volkart em $1960^{6}$ e que, em sua primeira concepção, considerava que a escolha do tratamento seria determinada por uma lógica de consumo, recaindo sobre aquele que apresentasse a melhor relação custo-benefício para o paciente. As teorias sobre este comportamento vão sofrer mudanças ao longo das décadas seguintes a partir de críticas a este modelo considerado utilitarista, e do reconhecimento de que as ações dos indivíduos na busca de cuidado eram interpretadas somente do ponto de vista de suas demandas por serviços do sistema de saúde e de uma crença inquestionável no modelo biomédico ${ }^{5}$. A inclu- são de fatores culturais, cognitivos e sociais na análise do comportamento sobre procura de serviços e a influência dos estudos sobre redes sociais ${ }^{7}$, imprimem uma nova perspectiva à interpretação deste comportamento. Os estudos etnográficos realizados na década de 70, a partir da observação de diferentes grupos sociais, prestam uma importante contribuição neste sentido ${ }^{4}$. O exemplo mais significativo deste esforço são os conceitos de Modelos Explicativos (Explanatory Models) e de Sistemas de Atenção à Saúde (Health Care System) desenvolvidos por A. Kleinman. Segundo este autor, Modelos Explicativos são as concepções sobre a enfermidade e as formas de tratamento empregadas por todos aqueles engajados em um processo clínico e que vão definir qual setor do Sistema de Atenção à Saúde será acionado no processo de cuidado. Sistemas de Atenção à Saúde são constituídos por três setores que caracterizam práticas de cuidado de naturezas diversas: o profissional, constituído pelas práticas formais de exercício da medicina (biomedicina, homeopatia, etc), o popular (cuidados caseiros, auto-cuidado) ou o folk (práticas místicas e religiosas de cuidados $)^{8}$.

Sobre o comportamento e fatores que influenciam a utilização de serviços de saúde, Biddle et al. observam que na vasta literatura existente, duas diferentes abordagens se destacam: uma, dominante e de cunho positivista, que procura caracterizar as diferenças entre usuários e não usuários através da definição de um "perfil do usuário” com padrões de comportamentos relativos ao uso do serviço e em termos de facilitadores ou barreiras (psicológicas, geográficas, estruturais, etc.) para o acesso, e outra, de caráter dinâmico, que trata a procura de serviços de saúde como um processo social não só influenciado pelos dispositivos biomédicos existentes mas também pelos recursos leigos e as redes sociais. Nessa abordagem o comportamento do enfermo é observado do ponto de vista de "como os sintomas são interpretados e gerenciados por indivíduos e pela comunidade" .

Os diversos enfoques possíveis na observação de itinerários terapêuticos podem subsidiar processos de organização de serviços de saúde e gestão, na construção de práticas assistenciais compreensivas e contextualmente integradas.

Os estudos e investigações sobre itinerários terapêuticos são relativamente recentes e apesar de sua potencialidade para a compreensão do comportamento em relação ao cuidado em saúde e ao uso de serviços, não tem expressão conhecida no Brasil. 
O objetivo deste trabalho é mapear o estado da arte da produção científica nacional acerca do tema itinerários terapêuticos no cuidado à saúde, nos últimos 20 anos, descrevendo e analisando as abordagens realizadas.

\section{Método}

Trata-se de um estudo descritivo a partir da revisão da produção científica desenvolvida no Brasil nos últimos 20 anos, que tem como núcleo de interesse a trajetória de pessoas em busca de atenção aos seus problemas de saúde. A coleta de dados foi realizada durante os meses de setembro a novembro de 2008, através dos portais da Biblioteca Virtual em Saúde (www.bireme.br). As bases de dados consideradas foram Lilacs (Literatura Latino-americana em Ciências da Saúde), Medline (Literatura Internacional em Ciências da Saúde) e Scielo (Scientific Electronic Library Online). Por falta de descritores que façam referência direta a este tema, o rastreamento inicial utilizou termos, nos idiomas português e inglês, que remetem diretamente ao objeto de interesse: itinerários terapêuticos; itinerários de cuidado; trajetórias terapêuticas; trajetórias de tratamento; trajetória do cuidado; trajetória do paciente; illness itineraries; therapeutic itineraries. Outros sinônimos foram utilizados mas sem resultados. Com o cruzamento de descritores comuns, identificados nos artigos localizados com estes termos, realizaramse novas buscas. São eles: antropologia; aceitação pelo paciente de cuidados de saúde; acesso aos serviços de saúde; tomada de decisão.

A seleção baseou-se nos seguintes critérios: artigos originais, publicados em periódicos indexados nas principais bases de dados em Ciências da Saúde, que relatam estudos realizados no Brasil nos últimos 20 anos, no período de janeiro de 1989 a dezembro de 2008 e cujo tema central fosse itinerários terapêuticos. Foram excluídos resenhas, editoriais, artigos de revisão e aqueles que focalizam a trajetória ou fluxo de pacientes em ambientes internos de serviços assistenciais.

Os trabalhos selecionados foram sistematizados em uma matriz composta das seguintes informações: Título, autor, ano de publicação e vinculação acadêmica; objetivos; tipo de estudo e métodos; principais resultados.

A análise do material teve como objetivo descrever as características dos estudos segundo as seguintes categorias: núcleo de interesse do estudo; abrangência do conceito de "itinerário terapêutico" utilizado; finalidade do estudo. A referência meto- dológica para a análise foi o método de Análise de Conteúdo em adaptação proposta por Bardin ${ }^{10}$.

\section{Resultados}

A busca inicial a partir dos termos diretamente relacionados ao tema resultou em 242 artigos. Do total, 158 artigos foram levantados na base de dados LILACS, 50 na Scielo, 20 na Medline, 14 na ADOLEC. Excluídas as repetições e os artigos que não preenchem os critérios estabelecidos para esta revisão, resultaram 13 artigos. Destes, dois foram excluídos por abordar a trajetória de vida do paciente a partir, exclusivamente, da sua percepção sobre a doença e não a trajetória de busca de cuidado. Foram, portanto, selecionados 11 artigos, três dos quais publicados em português e inglês. Os descritores comuns identificados nestes artigos, não mostraram sensibilidade à captação de nenhum outro trabalho além daqueles já localizados na primeira busca. Observou-se assim, a inexistência de palavras-chaves nos bancos de dados pesquisados, que tenham vinculação direta com esta temática. Os artigos selecionados são apresentados sinteticamente no Quadro 1. A caracterização dos artigos segundo as categorias propostas para análise é apresentada no Quadro 2.

\section{Discussão e considerações finais}

A leitura e análise de conteúdo dos 11 artigos foram norteadas pelas categorias apresentadas e descritas conforme o Quadro 2. Cada uma delas passa a ser explicada e discutida a seguir, à luz da literatura dos artigos analisados.

Os estudos sobre itinerários terapêuticos no Brasil são relativamente recentes. Este dado evidencia, conforme observado por Conill et al., que os arranjos estabelecidos por usuários na superação de problemas de acesso e integralidade expressam uma riqueza pouco aproveitada durante a formação de profissionais ou mesmo por pesquisadores e gestores ${ }^{19}$.

Com exceção de um artigo datado de 1993, todos os outros foram produzidos no período de 2000 a 2008.

Podemos pensar que o incremento neste período esteja relacionado à implantação de modelos assistenciais mais próximos à realidade local do paciente, influenciados pela ênfase no nível primário como eixo organizador da atenção, crescente a partir da década de 90 e, portanto, mais interessados em conhecer padrões de comportamento. 
Quadro 1. Artigos sobre estudos de itinerários terapêuticos:

\begin{tabular}{|c|c|c|c|c|}
\hline $\begin{array}{l}\text { Autor } \\
\text { e ano }\end{array}$ & Objetivos & $\begin{array}{c}\text { Desenho de estudo } \\
\text { e métodos }\end{array}$ & População & Principais resultados \\
\hline $\begin{array}{l}\text { Oliveira } \\
\text { DR et al., } \\
2003^{11}\end{array}$ & $\begin{array}{l}\text {. Conhecer o sistema de cuidado a } \\
\text { saúde percorrido pelo idoso renal } \\
\text { crônico } \\
\text {. Sensibilizar o enfermeiro para } \\
\text { ações de prevenção da doença renal } \\
\text { crônica }\end{array}$ & $\begin{array}{l}\text {. Qualitativo } \\
\text {. Entrevistas abertas } \\
\text {. Análise } \\
\text { interpretativa }\end{array}$ & $\begin{array}{l}\text { Seis idosos } \\
(>60) \text { em } \\
\text { clínica de TRS } \\
\text { - Curitiba - } \\
\text { PR }\end{array}$ & $\begin{array}{l}\text {. Itinerário de cuidado se dá de } \\
\text { acordo com significados } \\
\text { culturalmente construído } \\
\text {. Itinerário é construído } \\
\text { interligando os três setores de } \\
\text { atenção à saúde descritos por } \\
\text { Kleinman (1980) - profissional, } \\
\text { familiar e folk }\end{array}$ \\
\hline $\begin{array}{l}\text { Mattosinho } \\
\text { MMS, } \\
\text { Silva } \\
\text { DMGV et } \\
\text { al., } 2007^{12}\end{array}$ & $\begin{array}{l}\text {. Conhecer as práticas e o percurso } \\
\text { de cuidado do adolescente com } \\
\text { Diabetes Mellitus (DM) tipo } 1 \\
\text {. Conhecer como se dá a avaliação } \\
\text { do cuidado pelo paciente e família }\end{array}$ & $\begin{array}{l}\text { - Qualitativo } \\
\text { - Tipo: convergente } \\
\text { assistencial } \\
\text { - Entrevista } \\
\text { semiaberta } \\
\text { - Análise de } \\
\text { conteúdo temática }\end{array}$ & $\begin{array}{l}\text {. Cinco } \\
\text { dolescentes } \\
\text { com DM tipo } \\
1 \quad \text { residentes } \\
\text { em } \\
\text { Florianópolis - } \\
\text { SC } \\
\text { - Quinze } \\
\text { familiares }\end{array}$ & $\begin{array}{l}\text { - As formas como as pessoas } \\
\text { interpretam a doença determina } \\
\text { o cuidado a ser procurado } \\
\text {. Cuidado perpassa três setores de } \\
\text { atenção à saúde descritos por } \\
\text { Kleinman (1980) e não é estável } \\
\text {. Compreender o itinerário } \\
\text { terapêutico de adolescentes com } \\
\text { DM e seus familiares contribui } \\
\text { para ação humanizadora }\end{array}$ \\
\hline $\begin{array}{l}\text { Silva } \\
\text { DMGV et } \\
\text { al., } 2004^{13}\end{array}$ & $\begin{array}{l}\text { Identificar escolhas terapêuticas } \\
\text {. Identificar elementos do processo } \\
\text { de decisão e avaliação do cuidado e } \\
\text { tratamento por pessoas que vivem } \\
\text { com DPOC }\end{array}$ & $\begin{array}{l}\text { - Qualitativo } \\
\text { - Análise } \\
\text { interpretativa }\end{array}$ & $\begin{array}{l}12 \text { pessoas } \\
\text { com DPOC } \\
\text { internadas ou } \\
\text { em } \\
\text { tratamento } \\
\text { ambulatorial }\end{array}$ & $\begin{array}{l}\text {. Itinerário ocorre em etapas } \\
\text { sempre acompanhadas de } \\
\text { avaliações de decisões e de } \\
\text { resultados } \\
\text {. Predomínio do setor profissional } \\
\text { apesar dos setores popular e folk } \\
\text { também serem utilizado } \\
\text {. Não há linearidade no } \\
\text { tratamento da DPOC }\end{array}$ \\
\hline $\begin{array}{l}\text { Mussi FC } \\
\text { et al., } \\
2007^{14}\end{array}$ & $\begin{array}{l}\text {. Analisar Itinerários Terapêuticos } \\
\text { de pessoas que sofreram Infarto } \\
\text { Agudo do Miocárdio (IAM) } \\
\text {. Contribuir na qualificação do } \\
\text { acesso e do atendimento dos } \\
\text { serviços de urgência }\end{array}$ & $\begin{array}{l}\text { - Quali-quantitativo } \\
\text { - Entrevistas } \\
\text { - Análise de } \\
\text { Conteúdo } \\
\text { - Inferência } \\
\text { estatística }\end{array}$ & $\begin{array}{l}43 \text { mulheres e } \\
54 \text { homens } \\
\text { que sofreram } \\
\text { IAM com dor }\end{array}$ & $\begin{array}{l}\text {. } 28 \% \text { das pessoas optaram por } \\
\text { meios de transporte e locais de } \\
\text { atendimento inadequados } \\
\text {. Problemas de acesso } \\
\text { relacionam-se à falta de estrutura } \\
\text { dos serviços de atendimento } \\
\text {. Dificuldades para o diagnóstico } \\
\text { do IAM. } \\
\text { - Necessidade de programas de } \\
\text { educação para saúde direcionada } \\
\text { ao usuário e qualificação do } \\
\text { profissional para a assistência ao } \\
\text { IAM }\end{array}$ \\
\hline $\begin{array}{l}\text { Kovacs } \\
\text { MH et al., } \\
2005^{15}\end{array}$ & $\begin{array}{l}\text {. Conhecer a trajetória de criança } \\
\text { com demanda de urgência } \\
\text {. Caracterizar vínculo da criança } \\
\text { atendida na urgência, com o serviço } \\
\text { de atenção básica } \\
\text {. Verificar a adequação da } \\
\text { morbidade ao perfil organizacional } \\
\text { dos serviços. }\end{array}$ & $\begin{array}{l}\text { - Quanti-qualitativo } \\
\text { - Questionário } \\
\text { - Entrevista } \\
\text { semiaberta } \\
\text { - Exame clínico } \\
\text { - Análises } \\
\text { estatísticas } \\
\text { - Análises temáticas }\end{array}$ & $\begin{array}{l}\text { - Cinco } \\
\text { unidades } \\
\text { públicas de } \\
\text { referência para } \\
\text { urgência/ } \\
\text { emergência } \\
\text { pediátrica: } \\
\text { - } 383 \text { crianças } \\
\text { até cinco anos } \\
\text { e responsáveis }\end{array}$ & $\begin{array}{l}\text { - Apenas } 15,2 \% \text { dos agravos } \\
\text { demandados requeriam potencial } \\
\text { tecnológico condizente com a } \\
\text { capacidade assistencial } \\
\text {. Demanda espontânea decorre } \\
\text { da legitimidade dos serviços de } \\
\text { urgência perante a população } \\
\text {. Dificuldades de acesso e } \\
\text { resolubilidade da atenção básica } \\
\text { Indefinições sobre papel das } \\
\text { unidades básicas }\end{array}$ \\
\hline
\end{tabular}


Quadro 1. continuação

\begin{tabular}{|c|c|c|c|c|}
\hline $\begin{array}{l}\text { Autor } \\
\text { e ano }\end{array}$ & Objetivos & \begin{tabular}{|c|}
$\begin{array}{c}\text { Desenho de estudo } \\
\text { e métodos }\end{array}$ \\
\end{tabular} & População & Principais resultados \\
\hline $\begin{array}{l}\text { Scholze } \\
\text { AS, Silva } \\
\text { YF, 2005 }\end{array}$ & $\begin{array}{l}\text { - Analisar os riscos potenciais à } \\
\text { saúde em famílias com pessoas sob } \\
\text { cuidados domiciliários, observadas } \\
\text { por agentes comunitários de saúde } \\
\text { (ACS) }\end{array}$ & $\begin{array}{l}\text { - Qualitativo } \\
\text { entrevistas } \\
\text { observação de } \\
\text { ambientes, pessoas e } \\
\text { documentos } \\
\text { - Análise de } \\
\text { conteúdo temática } \\
\text { proposta por } \\
\text { Minayo (1988) }\end{array}$ & $\begin{array}{l}\text { Q Quatro } \\
\text { Agentes } \\
\text { Comunitários } \\
\text { de Saúde do } \\
\text { Programa } \\
\text { Saúde da } \\
\text { Família (PSF) } \\
\text { - Camboriú - } \\
\text { SC }\end{array}$ & $\begin{array}{l}\text {. Itinerários de cura e cuidado } \\
\text { determinados pelas limitações no } \\
\text { acesso aos serviços formais de } \\
\text { saúde } \\
\text { - Necessidade de estimulo a } \\
\text { autonomia e ao autocuidado dos } \\
\text { pacientes } \\
\text { - Necessidade de prevenção sobre } \\
\text { riscos potenciais nos itinerários, } \\
\text { mesmo que não incluam o } \\
\text { sistema de saúde formal. }\end{array}$ \\
\hline $\begin{array}{l}\text { Merino } \\
\text { MFGL, } \\
\text { Marcon } \\
\text { SS, } 2007^{17}\end{array}$ & $\begin{array}{l}\text { - Identificar diferenças de gênero } \\
\text { em comportamentos em saúde } \\
\text {. Conhecer o itinerário terapêutico } \\
\text { de adultos do município de Porto } \\
\text { Rico - PR }\end{array}$ & $\begin{array}{l}\text { - Qualitativo } \\
\text { - Entrevistas } \\
\text { semiestruturadas } \\
\text { - Análise de } \\
\text { conteúdo temática } \\
\text { proposta por } \\
\text { Minayo (1988) }\end{array}$ & $\begin{array}{l}29 \text { pessoas } 20- \\
59 \text { anos de } \\
\text { ambos os } \\
\text { sexos }\end{array}$ & $\begin{array}{l}\text {. Itinerário de homens e } \\
\text { mulheres é diferente } \\
\text {. Farmácia é primeira opção } \\
\text { homens e mulheres } \\
\text {. Esta opção } 3 \text { vezes maior entre } \\
\text { os homens. } \\
\text {. Utilização da rede formal é } \\
\text { maior e mais precoce por } \\
\text { mulheres } \\
\text {. O autocuidado (setor informal) } \\
\text { foi relatado por quase todos os } \\
\text { entrevistados }\end{array}$ \\
\hline $\begin{array}{l}\text { Silva DGV } \\
\text { et al., } \\
2006^{18}\end{array}$ & $\begin{array}{l}\text { - Conhecer o itinerário terapêutico } \\
\text { de pessoas com Diabetes Mellitus } \\
\text { (DM) tipos } 1 \text { e } 2 \text { nos diferentes } \\
\text { subsistemas de cuidado a saúde. }\end{array}$ & $\begin{array}{l}\text { - Qualitativo } \\
\text { - Entrevista aberta } \\
\text { - Grupo focal } \\
\text {. Análise } \\
\text { interpretativa com } \\
\text { triangulação }\end{array}$ & $\begin{array}{l}29 \text { pessoas } \\
\text { com DM }>18 \\
\text { anos } \\
\text { acompanhadas } \\
\text { em } \\
\text { instituições de } \\
\text { saúde }\end{array}$ & $\begin{array}{l}\text { O subsistema profissional: } \\
\text { referência inicial na busca da } \\
\text { compreensão sobre o que estava } \\
\text { acontecendo } \\
\text { - Após diagnóstico, diferentes } \\
\text { tratamentos e cuidados. } \\
\text { - Percurso não é linear. } \\
\text { Mudanças de trajetórias são } \\
\text { frequentes }\end{array}$ \\
\hline $\begin{array}{l}\text { Conill EM } \\
\text { et al., } \\
2008^{19}\end{array}$ & $\begin{array}{l}\text { Estudo de itinerários terapêuticos } \\
\text { de usuários de serviços de saúde } \\
\text { suplementar através de condições } \\
\text { marcadoras (IAM, CA mama, } \\
\text { alcoolismo e parto) no município } \\
\text { de Florianópolis. }\end{array}$ & $\begin{array}{l}\text { - Qualitativo } \\
\text { - Mapeamento } \\
\text { exploratório através } \\
\text { de situações } \\
\text { marcadoras } \\
\text { - Entrevistas } \\
\text { - Análise de } \\
\text { conteúdo temática }\end{array}$ & $\begin{array}{l}.17 \text { usuários } \\
\text { com história } \\
\text { de IAM } \\
\text {. } 11 \text { mulheres } \\
\text { com câncer de } \\
\text { mama } \\
.10 \text { usuários } \\
\text { com história } \\
\text { de alcoolismo } \\
\text {. } 11 \text { mulheres } \\
\text { com histórico } \\
\text { de parto até } \\
\text { um ano e } \\
\text { meio }\end{array}$ & $\begin{array}{l}\text {. Percursos influenciados pela } \\
\text { cobertura dos planos de saúde e } \\
\text { pela disponibilidade (ou não) } \\
\text { desses recursos } \\
\text {. Parte dos usuários procuram } \\
\text { recursos dos três subsistemas - o } \\
\text { profissional (incluindo o mix } \\
\text { público-privado), o informal e o } \\
\text { popular (significativos nas áreas } \\
\text { da saúde mental e oncologia) } \\
\text {. Diversos arranjos na superação } \\
\text { de lacunas do acesso e da } \\
\text { integralidade da atenção. } \\
\text { - abordagem socioantropológica é } \\
\text { útil para compreensão do } \\
\text { significado de público e privado } \\
\text { no sistema de saúde, bem como } \\
\text { dos modelos de cuidados } \\
\text { empreendidos pelos usuários. }\end{array}$ \\
\hline
\end{tabular}




\begin{tabular}{|c|c|c|c|c|c|}
\hline \multirow[t]{4}{*}{ 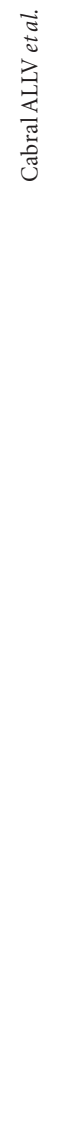 } & Quadro 1. & ntinuação & & & \\
\hline & $\begin{array}{l}\text { Autor } \\
\text { e ano }\end{array}$ & Objetivos & $\begin{array}{c}\text { Desenho de estudo } \\
\text { e métodos }\end{array}$ & População & Principais resultados \\
\hline & $\begin{array}{l}\text { Rabelo } \\
\text { MC, } \\
1993^{20}\end{array}$ & $\begin{array}{l}\text { Contribuir para o entendimento } \\
\text { das formas pelas quais as visões de } \\
\text { mundo e projetos de cura de } \\
\text { diferentes cultos religiosos são de } \\
\text { fato incorporados à experiência } \\
\text { cotidiana de doentes mentais e seus } \\
\text { familiares. }\end{array}$ & $\begin{array}{l}\text { Qualitativo } \\
\text {. Reconstituição de } \\
\text { trajetória de cura. } \\
\text { - Análise } \\
\text { interpretativa }\end{array}$ & $\begin{array}{l}\text { - Adolescente } \\
\text { portadora de } \\
\text { sofrimento } \\
\text { mental }\end{array}$ & $\begin{array}{l}\text {. Relação entre símbolos } \\
\text { religiosos e vida social não é } \\
\text { definida a priori, mas estabelecida } \\
\text { no curso de eventos concretos } \\
\text { nos quais os indivíduos se } \\
\text { apropriam, confrontam e } \\
\text { reinterpretam os símbolos à luz } \\
\text { de determinados fins e interesses. }\end{array}$ \\
\hline & $\begin{array}{l}\text { Gerhardt } \\
\text { TE, } 2006^{21}\end{array}$ & $\begin{array}{l}\text {. Geral: Associação entre situações } \\
\text { de vida, pobreza e saúde em } \\
\text { Paranaguá-PR } \\
\text {. Específico (artigo): Estudo dos } \\
\text { itinerários terapêutico de famílias } \\
\text { de baixa renda }\end{array}$ & $\begin{array}{l}\text { - Qualitativo } \\
\text { - Entrevistas semi- } \\
\text { aberta } \\
\text { - Observação } \\
\text { participante } \\
\text { - Análise de } \\
\text { conteúdo }\end{array}$ & $\begin{array}{l}75 \text { famílias } \\
\text { de baixa renda } \\
\text { da Ilha de } \\
\text { Valadares e } \\
\text { Franjas } \\
\text { (Paranaguá) }\end{array}$ & $\begin{array}{l}\text { - Indivíduos constroem } \\
\text { estratégias de vida de acordo com } \\
\text { suas capacidades, histórias de vida } \\
\text { e experiências. } \\
\text { - Condições de vida e estado de } \\
\text { saúde: nem sempre possui } \\
\text { determinação direta. } \\
\text {. Utilização de terapêuticas } \\
\text { médicas e não médicas (caseiras) } \\
\text { no enfrentamento das situações } \\
\text {. Estratégias de enfrentamento } \\
\text { compreendem relações familiares, } \\
\text { vizinhança e outras redes } \\
\text { solidárias. }\end{array}$ \\
\hline
\end{tabular}

Quadro 2. Características dos estudos sobre itinerários terapêuticos identificados a partir da análise dos artigos selecionados.

\begin{tabular}{|c|c|c|}
\hline Categoria & Característica do estudo & Estudos identificados * \\
\hline \multirow{3}{*}{$\begin{array}{l}\text { Núcleo de interesse } \\
\text { destacado na abordagem } \\
\text { sobre Itinerários } \\
\text { Terapêuticos }\end{array}$} & $\begin{array}{l}\text { Comportamento/percepção paciente ou família sobre } \\
\text { doença ou tratamento }\end{array}$ & $11,12,13,17,18,20,21$ \\
\hline & Organização/utilização do serviço de saúde & 14,15 \\
\hline & $\begin{array}{l}\text { Reflexão sobre acesso/ organização de serviços associada à } \\
\text { reflexão sobre percepção e comportamento do paciente }\end{array}$ & 19,16 \\
\hline \multirow{2}{*}{$\begin{array}{l}\text { Abrangência do Conceito } \\
\text { de Itinerário Terapêutico } \\
\text { utilizado }\end{array}$} & Restrito ao sistema de saúde formal & 14,15 \\
\hline & $\begin{array}{l}\text { Considera todas as práticas implicadas na recuperação da } \\
\text { saúde }\end{array}$ & $11,12,13,16,17,18,19,20,21$ \\
\hline \multirow[t]{4}{*}{ Finalidade do estudo } & Recomendar a Adequação do perfil profissional & $11,12,14$ \\
\hline & Recomendar adequação da rede assistencial & $14,15,19$ \\
\hline & Recomendar adoção de programas de educação em saúde & 14,16 \\
\hline & Conhecer comportamento e ou realidade do paciente & $11,13,17,18,20,21$ \\
\hline
\end{tabular}

* Referência bibliográfica.

Antes de discutir os artigos propriamente ditos, é importante observar que a antropologia aplicada à área de saúde no Brasil, configura-se muito mais no campo da antropologia social do que no campo da antropologia médica, pois não se desenvolveu subordinada ao campo da medicina, mas sim junto a várias disciplinas que configuram o campo das ciências sociais em saúde, como a sociologia e as ciências políticas ${ }^{22}$. No entanto, a principal influência identificada na 
produção científica nacional sobre itinerários terapêuticos decorre dos modelos desenvolvidos no âmbito da segunda corrente.

O núcleo de interesse predominante entre os estudos refere-se às percepções do paciente sobre a doença e a influência destas sobre seu comportamento em relação a procura de atenção e ao tratamento propriamente dito ${ }^{23}$. Estão incluídos aqui, os estudos que utilizam a concepção de Sistema de Atenção à Saúde e o conceito de Modelos Explicativos desenvolvidos por Kleinman sob influência da antropologia interpretativa de Geertz ${ }^{24}$. Em geral apresentam um enfoque microssociológico, comunitário ou de grupo, sem aprofundar questões sobre contextos socioeconômicos ou organização de serviços de saúde. A população de interesse destes estudos é composta, principalmente, por portadores de doenças crônicas como diabetes mellitus, doença renal crônica, asma e problemas psiquiátricos que, invariavelmente, necessitam de acompanhamento contínuo ${ }^{11-13,17,18,20}$. Têm como importantes objetivos, entre outros, conhecerem os dispositivos de cuidados acionados pelo paciente e pela família no enfrentamento da doença e sugerir o olhar ampliado por parte dos profissionais de saúde sobre o universo cultural dos usuários, de forma a adequar práticas e a atingir resultados terapêuticos mais efetivos.

Outra linha de estudos sobre itinerários utiliza este conhecimento como suporte para a avaliação da efetividade das redes de serviços na garantia do acesso e para a detecção de necessidades a serem consideradas no desenvolvimento de programas educativos em saúde, capacitação de profissionais e adequação de fluxos. Aqui, a trajetória e o comportamento do paciente são observados a partir do ponto de vista do sistema de serviços de saúde e das práticas biomédicas desenvolvidas por estes. O foco destes trabalhos são os atendimentos de urgência em unidade de pronto socorro. São estudos que associam recursos metodológicos quantitativos e qualitativos buscando identificar hiatos nos fluxos formais de atendimento com a intenção de corrigilos e de garantir, através de práticas informativas, um melhor direcionamento dos pacientes por entre estes fluxos ${ }^{14,15}$. Têm objetivos pragmáticos de imprimir agilidade na identificação e na solução de problemas.

Outras abordagens incorporam as duas perspectivas anteriores quando, além do reconhecimento dos diferentes sistemas de atenção à saúde, consideram na leitura dos itinerários que os processos saúde/doença/cuidado estão inseridos em um macro-contexto determinado por fatores econômicos e sociais, os quais envolvem também uma dimensão subjetiva e cultural que vai interferir na forma como as pessoas utilizam os serviços formais de saúde. Expressam a preocupação em conhecer as iniciativas dos pacientes dentro de um campo de possibilidades, geradas por situações concretas de dificuldade ou por facilidade de acesso ou incapacidade do serviço em atender determinadas demandas. Neste sentido, Conill et al., em estudo multicêntrico sobre beneficiários do sistema suplementar de saúde reconstitui itinerários a partir de quatro situações marcadoras (parto, alcoolismo, infarto agudo do miocárdio e câncer de mama), levantando informações sobre as experiências vividas pelos usuários nos serviços e quais arranjos estabelecidos por eles para cada uma destas situações. Os autores consideram que estes arranjos incluem, além do serviço suplementar, o público e outros sistemas de atenção. $\mathrm{Na}$ análise das estratégias utilizadas pelos usuários na construção do cuidado, articulam elementos da percepção sobre a doença, da política de saúde e da gestão de serviços ${ }^{19}$.

Scholze e Silva, também nesta direção, investigaram riscos potenciais à saúde em itinerários de cura e cuidado através das narrativas de Agentes Comunitários de Saúde do Programa de Saúde da Família sobre visitas a pacientes domiciliares. $\mathrm{O}$ estudo problematiza o autocuidado e o cuidado familiar enquanto estratégias de enfrentamento dos problemas colocados pela falta de acesso ao serviço de saúde. Discute o risco potencial destas práticas, mesmo reconhecendo sua legitimidade. Enfatizam a necessidade do desenvolvimento de estratégias assistenciais e de educação em saúde que considerem esta realidade. Aqui há uma opção em adotar a expressão "itinerários de cura e de cuidado" em lugar de "itinerários terapêuticos", como forma de enfatizar o aspecto participativo e dinâmico de redes sociais e de familiares na produção do cuidado ${ }^{16}$.

Gerhardt, em estudo desenvolvido em Paranaguá - PR, único do gênero, investiga a relação entre itinerários terapêuticos e pobreza. Diferentemente dos outros estudos, desloca o foco de interesse da percepção individual ou de grupo sobre a doença, para o campo da desigualdade social e diversidade cultural, que se reflete na busca do cuidado. Pressupõe que "para se compreender a saúde dos indivíduos e a forma como eles enfrentam a doença torna-se necessário analisar suas práticas (itinerários terapêuticos), a partir do contexto onde elas tomam forma" ${ }^{21}$. Trabalhando, segundo Minayo, com referenciais am- 
pliados e contextualizados, a autora inscreve sua análise no campo da antropologia social. Neste sentido agrega à leitura sobre a busca de atenção outros fatores além dos elementos da cultura. Utiliza a definição de Massé, para quem "a procura de cuidados está condicionada tanto pelas atitudes, valores e ideologias quanto pelos perfis da doença, pelo acesso econômico e pela disponibilidade de tecnologia"25. Gerhardt endossa a crítica tecida por Alves sobre o conceito de Modelos Explicativos quando considera que este, apesar da influência da hermenêutica na sua concepção, apresenta caráter muito mais explicativo do que interpretativo e termina por reduzir as ações dos indivíduos à lógica inerente a esses modelos ${ }^{21,26}$.

As diferentes formas de utilização do conhecimento sobre itinerários terapêuticos identificadas nos artigos levantados expressam a versatilidade deste objeto que pode atender a diferentes interesses com um objetivo comum: a qualificação do cuidado prestado.

Apesar de não haver um consenso em relação aos conceitos de Modelos Explicativos e Sistemas de Atenção à Saúde desenvolvidos por Kleinman, estes ocupam na literatura pesquisada um importante papel de referência na explicação de comportamentos de procura de cuidados e dos itinerários percorridos. Percebe-se em algumas das experiências apresentadas um interessante movimento de adaptação do uso destes conceitos de forma a torná-los menos herméticos e previsíveis e mais permeáveis às influências de fatores contextuais, constituindo-se em ferramenta de valor para a compreensão de determinados comportamentos em relação ao processo saúde/doença/ cuidado, em determinadas circunstâncias ${ }^{16,19,21}$.

É inegável a contribuição da abordagem socioantropológica, em seus diferentes matizes conceituais, naqueles estudos onde os itinerários são tratados a partir de uma perspectiva ampliada que associa a percepção do paciente à análise do contexto real onde se inscrevem as suas práticas de cuidado. Tais estudos mostram ainda que itinerários terapêuticos não são exclusivamente determinados por contingências relacionadas a facilidades ou dificuldades no acesso, em uma relação de consumo com os serviços de saúde.

Não obstante, observa-se que a literatura sobre itinerários terapêuticos é ainda limitada em relação às possibilidades que o estudo destes oferece. Outros núcleos de interesse, além daqueles aqui identificados, podem ser estabelecidos ou aprofundados atendendo às necessidades de reflexão colocadas atualmente pelo campo do cuidado em saúde.

A opção por desenhos assistenciais centrados no usuário e em seu território coloca à gestão em saúde o desafio de conhecer mais profundamente as características e os determinantes da busca de cuidado. Mais do que saber, como nos estudos de demanda, se essa busca acontece ou não, é importante conhecer, conforme Biddle et al., como e em que momento se dá e quais os significados atribuídos pelo usuário9. Neste sentido, a análise de itinerários terapêuticos pode ser uma estratégia complementar aos estudos quantitativos desenvolvidos com a finalidade de descrever o perfil do usuário ou padrões de utilização de serviços de saúde.

É importante observar que a inexistência de descritores mais precisos sobre este tema nos bancos de dados pesquisados, pode ter provocado a exclusão de artigos não detectados pela estratégia de busca utilizada nesta revisão. Ainda assim, os trabalhos localizados demonstram a pertinência dos estudos sobre itinerários, tanto para a reflexão sobre as relações entre profissional e paciente quanto para as atividades de planejamento e gestão. 


\section{Colaboradores}

ALLV Cabral participou do planejamento e elaboração do estudo, pesquisa bibliográfica, avaliação metodológica e redação do artigo. A Martínez-Hernáez: participou do planejamento e elaboração do estudo, revisão metodológica e redação final do artigo. EIG Andrade participou da avaliação metodológica e da redação final do artigo. ML Cherchiglia participou do planejamento e da elaboração do estudo, da avaliação metodológica e da redação final do artigo.

\section{Agradecimentos}

Esse estudo foi financiado parcialmente pela Fundação de Amparo à Pesquisa do Estado de Minas Gerais - FAPEMIG e pelo Fundo Nacional de Saúde/Ministério da Saúde.

\section{Referências}

1. Silva LMV, Formigli VLA, Cerqueira MP, Kruchevsky L, Teixeira MMA, Barbosa ASM, Conceiçäo PSA, Khouri MA, Nascimento CL. O processo de distritalização e a utilização de serviços de Saúde Avaliação do caso de Pau da Lima, Salvador, Bahia, Brasil. Cad Saude Publica 1995; 11(1):72-84.

2. Goldbaum M, Gianini RJ, Novaes HMD, César CLG. Utilização de serviços de saúde em áreas cobertas pelo programa saúde da família (Qualis) no Município de São Paulo. Rev Saude Publica 2005; 39(1):9099.

3. Travassos C, Viacava F, Pinheiro R, Brito A. Utilização dos serviços de saúde no Brasil: gênero, características familiares e condição social. Rev $\mathrm{Pa}$ nam Salud Publica 2002; 11(5/6):365-373.

4. Alves PCB, Souza IM. Escolha e avaliação de tratamento para problemas de saúde: considerações sobre o itinerário terapêutico. In: Rabelo MC, Alves PCB, Souza IMA, organizadores. Experiência de doença e narrativa. Rio de Janeiro: Editora Fiocruz; 1999. p. 125-38.

5. Martinez HA. Os itinerários terapêuticos e a relação médico-paciente. Universitat Rovira i Virgili. Tradução de Virgínia Jorge Barreto. Belo Horizonte, Abril 2006. [texto de apoio]

6. Mechanic D, Volkart H. Stress, illness behavior, and the sick role. American Sociological Review 1961; 26(1):51-58.

7. Uchoa E, Vidal JM. Antropologia médica: elementos conceituais e metodológicos para uma abordagem da saúde e da doença. Cad Saude Publica 1994; 10(4):497-504.

8. Kleinman A. Patients and healers in the context of culture. California: Regents; 1980.

9. Bidlle L, Donovan J, Sharp D, Gunnell D. Explaining non-help-seeking amongst young adults with mental distress: a dynamic interpretive model of illness behavior. Sociology of Health \& Illness 2007; 29(7):983-1002.

10. Triviños ANS. Introdução à pesquisa em ciências sociais: a pesquisa qualitativa e educação. $1^{\text {a }}$ ed. São Paulo: Editora Atlas; 1987.

11. Oliveira DR, Lenardt MH, Tuoto FSl. O Idoso e o Sistema de Cuidado à Saúde na Doença Renal. Acta Pau. Enf 2003; 16(4):49-58.

12. Mattosinho MMS, Silva DMGV. Itinerário terapêutico do adolescente com Diabetes Mellitus tipo 1 e seus familiares. Rev Latino-am Enfermagem 2007; 15(6):1113-1119.

13. Silva DMGV, Meirelles BHS, Souza SS, Francioni F. O itinerário terapêutico de pessoas com problemas respiratórios crônicos. Texto Contexto Enferm 2004; 13(1):50-56.

14. Mussi FC, Passos LCS, Menezes, AA, Carameli, B. Entraves No Acesso à Atenção Médica: Vivências de pessoas com infarto agudo do miocárdio. Rev Assoc Med Bras 2007; 53(3):234-239.

15. Kovacs MH, Feliciano KVO, Sarinho SW, Veras AMCA. Acessibilidade às ações básicas entre crianças atendidas em serviços de pronto-socorro. Jornal de Pediatria 2005; 81(3):251-258. 
16. Scholze AS, Silva YF. Riscos Potenciais à Saúde em Itinerários de Cura e Cuidado. Cogitare Enferm 2005; 10(2):9-16.

17. Merino MFGL, Marcon SS. Concepções de saúde e itinerário terapêutico adotado por adultos de um município de pequeno porte. Rev Bras Enferm 2007; 60(6):651-658.

18. Silva DGV, Souza SS, Francione FF, Mattosinho MMS, Coelho MS, Sandoval RCB, Cunha MA, Ferreira N. Pessoas com Diabetes Mellitus: suas escolhas de cuidados e tratamentos. Rev Bras Enferm 2006; 59(3):297-302.

19. Conill EM, Pires D, Sisson MC, Oliveira, MC, Boing AF, Fertonani HP. O mix público-privado na utilização de serviços de saúde: um estudo dos itinerários terapêuticos de beneficiários do segmento de saúde suplementar brasileiro. Cien Saude Colet 2008; 13(5):1501-1510.

20. Rabelo, MC. Religião e Cura: Algumas Reflexões Sobre a Experiência Religiosa das Classes Populares Urbanas. Cad Saude Publica 1993; 9(3):316-325.

21. Gerhardt TE. Itinerários terapêuticos em situações de pobreza: diversidade e pluralidade. Cad Saude Publica 2006; 22(11):2449-2463.

22. Minayo MCS. Contribuições da antropologia para pensar a saúde. In: Campos GVS, Minayo MCS, Akerman M, Drumond Junior, Carvalho YM, organizadores. Tratado de saúde coletiva. São Paulo: Hucitec; 2006. p. 201-230.

23. Helman CG. Cultura, saúde e doença. Porto Alegre: Artes Médicas; 1994.

24. Geertz, C. A interpretação das culturas. Rio de Janeiro: Zahar; 1978.

25. Massé R. Culture et santé publique. Les contributions de l'anthropologie à la prévention et à la promotion de la santé. Montréal: Gaëtan Morin Éditeur; 1995.

26. Alves PC. A experiência da enfermidade: considerações teóricas. Cad Saude Publica 1993; 9(3):263-271.

Artigo apresentado em 12/07/2009

Aprovado em 10/10/2009

Versão final apresentada em 15/10/2009 